\title{
Gleason Score Correlation Between Prostate Biopsy and Radical Prostatectomy Specimens
}

\author{
(1) Erdem Öztürk MD, (1) Taha Numan Yıkılmaz MD \\ University of Health Sciences, Dr. Abdurrahman Yurtaslan Ankara Oncology Training and Research Hospital, Clinic of Urology, Ankara, Turkey
}

\begin{abstract}
Objective: Prostate cancer is the most common malignancy in men and the second cause of cancer-related mortality. Prostate biopsy and the Gleason score guide treatment decisions in prostate cancer. Several studies have investigated the correlation between biopsy scores and radical prostatectomy specimen scores. We also evaluated the correlation of Gleason scores of these specimens in our patient series.

Materials and Methods: We retrospectively reviewed the data of 468 men who were diagnosed with prostate cancer and underwent radical prostatectomy between 2008 and 2017. Patients' age, prostate-specific antigen levels at diagnosis, and prostate biopsy and radical prostatectomy specimen Gleason scores were recorded. Upgrading and downgrading were defined as increase or decrease of Gleason score of radical prostate specimen compared to Gleason score of prostate biopsy.

Results: A total of 442 men diagnosed with prostate cancer were included in the study. The mean age of the patients was $62.62 \pm 6.26$ years (44-84 years) and mean prostate specific antigen level was $9.01 \pm 6.84 \mathrm{ng} / \mathrm{mL}(1.09-49 \mathrm{ng} / \mathrm{mL})$. Prostate biopsy Gleason score was $<7$ in $335(75.8 \%) \mathrm{men}$, 7 in $80(18.1 \%)$ men, and $>7$ in $27(6.1 \%)$ men. Radical prostatectomy specimen Gleason score was $<7$ in $267(60.4 \%)$ men, 7 in $113(25.5 \%)$ men and $>7$ in 62 (14\%) men. Gleason correlation was highest in the 240 patients (71.6\%) with score $<7$ and was lowest in the 31 (38.75\%) patients with score $=7$.
\end{abstract}

Conclusion: This study demonstrated that the discordance rate between Gleason scores of prostate biopsy and radical prostatectomy specimens was $35.7 \%$.

Keywords: Prostate biopsy, radical prostatectomy, Gleason score

\section{Introduction}

Prostate cancer is currently one of the most common malignancies in men and the second most common cause of cancerspecific mortality after lung cancer (1). Diagnosis is based on digital rectal examination, serum prostate specific antigen (PSA) measurement, and when deemed necessary, transrectal prostate needle biopsy. Prostate needle biopsy provides information about tumor pathology, and therefore has a substantial impact on treatment decisions.

In spite of its importance in diagnosis and treatment planning, prostate needle biopsy may yield different Gleason scores than those determined by examination of radical prostatectomy
(RP) specimens. Despite the integration of recently developed imaging systems into prostate needle biopsy procedures and a higher number of biopsy cores being acquired, there is still considerable inconsistency in biopsy and RP specimen grading. Correlation between Gleason scores obtained from biopsy and RP specimens has been reported in the range of $41.3-63 \%$, with scores increasing in $21.9-47.4 \%$ of the patients and decreasing in $5-20.7 \%$ of the patients after RP $(2,3,4,5)$.

Gleason scores are important in terms of disease course and treatment planning. The aim of the present study was to determine concordance between Gleason scores obtained from prostate needle biopsy and RP specimens. 


\section{Materials and Methods}

A total of 468 patients who were diagnosed with prostate cancer by needle biopsy and underwent RP in our clinic between 2008 and 2017 were included in the study. Patients with incomplete data were excluded. The patients' age, pre-biopsy PSA values, biopsy pathology results, and post-RP pathology results were obtained retrospectively by medical record review.

Prostate biopsy was recommended for patients with suspicious digital rectal examination and/or elevated PSA ( $\geq 4 \mathrm{ng} / \mathrm{mL})$. All prostate biopsies were performed as transrectal ultrasoundguided biopsy using an 18-gauge, $200 \mathrm{~mm}$ biopsy needle. All patients in the study underwent 12-core prostate biopsy. Patients who were diagnosed with prostate cancer according to these biopsy results and underwent RP were included in the study.

The biopsy and RP specimens of the patients were evaluated by pathologists and all specimens were scored according to the Gleason grading system. Ensuring that there were sufficient numbers in each group, the patients were divided into 3 groups based on Gleason score $(<7,=7$, and $>7)$. Within each group, patients' biopsy and RP specimen Gleason scores were compared. A second analysis was done by separating patients with a Gleason score of 7 into two groups: $3+4$ and $4+3$. Cases were classified as upgrade if the RP specimen score was higher than the biopsy score, and as downgrade if RP score was lower than biopsy score.

\section{Results}

Of the total 468 patients who underwent RP, 442 met the inclusion criteria of the study. The mean age of the patients was 62.62 \pm 6.26 years (44-84 years) and their mean PSA value at time of diagnosis was $9.01 \pm 6.84 \mathrm{ng} / \mathrm{mL}(1.09-49 \mathrm{ng} /$ $\mathrm{mL})$. Gleason score based on prostate biopsy result was $<7$ for 335 patients (75.8\%), 7 for 80 patients (18.1\%), and $>7$ for 27 patients (6.1\%). Gleason score based on RP specimen pathology result was <7 for 267 patients (60.4\%), 7 for 113 patients (25.5\%), and $>7$ for 62 patients (14\%) (Table 1).

According to biopsy results, Gleason $3+3$ was the most observed pathology (75.8\%), followed by Gleason 3+4 (14\%). Similarly, according to RP specimen, the prevalence of Gleason $3+3$ was $60.4 \%$ and that of Gleason $3+4$ was $19.9 \%$. In 284 patients $(64.2 \%)$, biopsy results were similar to RP specimen results, whereas Gleason grade was downgraded in 39 patients (8.8\%) and upgraded in 119 patients (26.9\%). The highest compatibility was observed in the Gleason $<7$ patient group, which consisted of 240 patients (71.6\%). The lowest compatibility was observed in Gleason 7 patient group, which consisted of 31 patients (38.75\%). Although the total Gleason score remained constant, the results of 5 patients with biopsy results of Gleason 3+4 were changed to Gleason 4+3, and Gleason $4+3$ score was changed to Gleason $3+4$ in 7 patients (Table 2).

According to the $\mathrm{D}^{\prime}$ amico risk classification, patients were analyzed in subgroups of Gleason score $<7,=7$, and $>7$. Of 335 patients in the Gleason $<7$ group, 95 (39.5\%) were upgraded

\begin{tabular}{|c|c|}
\hline & $\begin{array}{l}\text { Number } \\
(n=442)\end{array}$ \\
\hline Age (years) & $62.6 \pm 6.2$ \\
\hline PSA (ng/mL) & $9.0 \pm 6.84$ \\
\hline $\begin{array}{l}\text { Biopsy Gleason scores } \\
3+3 \\
3+4 \\
4+3 \\
4+4 \\
3+5 \\
5+3 \\
4+5 \\
5+4 \\
5+5\end{array}$ & $\begin{array}{l}335 \\
62 \\
18 \\
17 \\
3 \\
1 \\
1 \\
3 \\
2\end{array}$ \\
\hline $\begin{array}{l}\text { Radical prostatectomy specimen Gleason scores } \\
3+3 \\
3+4 \\
4+3 \\
4+4 \\
3+5 \\
5+3 \\
4+5 \\
5+4 \\
5+5\end{array}$ & $\begin{array}{l}267 \\
88 \\
25 \\
42 \\
9 \\
0 \\
2 \\
6 \\
3\end{array}$ \\
\hline
\end{tabular}

Table 2. Change in Gleason scores between biopsy and radical prostatectomy specimens

\begin{tabular}{|l|l|l|l|}
\hline Biopsy score & Downgrade & Compatible & Upgrade \\
\hline$<7$ & & 240 & 95 \\
\hline$=7$ & 27 & 31 & 22 \\
$3+4$ & 22 & 21 & 19 \\
$4+3$ & 5 & 10 & 3 \\
\hline$>7$ & 12 & 13 & 2 \\
\hline Total & $39(8.8 \%)$ & $284(64.25 \%)$ & $119(26.9 \%)$ \\
\hline
\end{tabular}

after RP. Among the 80 patients with a biopsy Gleason score of 7 , results obtained from the two specimens were compatible in 31 (38.75\%), while 27 patients (33.75\%) were downgraded and 22 patients $(27.5 \%)$ were upgraded. Of the 27 patients with a biopsy score $>7,12$ patients (44.4\%) were downgraded.

\section{Discussion}

In prostate cancer, identifying Gleason score is the key factor in choosing from a broad spectrum of treatment options ranging from watchful waiting to multimodal treatment. In this widely used grading system, the first and second most common glandular patterns are identified and patients are placed into risk groups according to the sum of these two patterns (6). Gleason score determined from RP specimens is shown to be one of the predictive factors of patient survival (7). Although effective for predicting prognosis and making treatment decisions, Gleason scores may differ between prostate needle biopsy and RP specimen. In 2005, the International Society of Urological Pathology published a consensus report aiming to improve biopsy results and standardize biopsy technique. The systematic procedure for prostate biopsy described in that 
report led to better agreement between the results of biopsy and RP specimens (8).

There are several studies in literature regarding concordance between biopsy specimens and RP specimens in prostate cancer patients. Cookson et al. (9) reported a compatibility rate of $31 \%$ between scores of biopsy and RP specimens. They determined a biopsy score upgrade rate of $54 \%$ and a downgrade rate of $15 \%$. In another study, San Francisco et al. (10) reported a $67 \%$ compatibility rate between biopsy scores and surgical pathology scores, while biopsy scores were downgraded in $11 \%$ of the patients and upgraded in $22 \%$. This discrepancy between scores obtained from biopsies and surgical specimens has been the focus of numerous studies. In studies further evaluating patients with incompatible results, the upgrade rate has varied between $21.9-47.4 \%$ while the downgrade rate varies between $5-20.7 \%(3,4)$. The compatibility rate in our series was $64.2 \%$ and upgrade/downgrade rates were $26.9 \%$ and $8.8 \%$, respectively. Our data were consistent with the literature, though we observed slightly higher agreement between the two specimens.

Stav et al. (11) reported that agreement was lower in patients with Gleason scores between 2-4, and $94.2 \%$ of these patients were upgraded. In a study by Capitanio et al. (12) investigating 301 prostate cancer patients in the low-risk group according to D'amico classification, $38.5 \%$ of the patients were upgraded. According to the subgroup analysis performed in our study, of 335 patients with Gleason score of 6, biopsy and RP specimen grading was compatible in $240(71.6 \%)$, while scores were upgraded for the remaining 95 patients (28.4\%).

Donohue et al. (13) reported that $45 \%$ of patients with biopsy scores of 8-10 were downgraded. In a study of patients with Gleason score 9-10, D'elia et al. (14) reported that 58\% had compatible biopsy and RP specimen scores. In the present study, of the 25 patients with biopsy Gleason score $>7,12$ patients (44.4\%) were downgraded after RP, whereas $55.6 \%$ of the patients had compatible results.

D'elia et al. (14) also grouped and analyzed prostate cancer patients according to their Gleason score. Biopsy and RP specimen scores were similar for $57.4 \%$ of the patients in the Gleason $3+4$ group, whereas $6.4 \%$ of the patients were downgraded and $36.2 \%$ of patients were upgraded after RP. The same analysis in our patient group revealed compatible scores in $33.8 \%$, downgrade in $35.4 \%$, and upgrade in $30.6 \%$. D'elia performed a similar analysis with Gleason $4+3$ patients and determined compatibility, downgrade, and upgrade rates of $35.3 \%, 23.5 \%$, and $41.2 \%$, respectively, in that group. In our study, these rates were $55.5 \%, 27.7 \%$, and $16.6 \%$, respectively, in our Gleason 4+3 group.

\section{Study Limitations}

Limitations of this study include its retrospective design, the use of ultrasound guidance for biopsy, and the fact that the biopsy and surgical specimens were examined by different pathologists. Magnetic resonance imaging-guided biopsy and the pathological examinations by the same pathologist would likely increase the compatibility between the specimens.

\section{Conclusion}

Gleason score is the most important parameter in prostate cancer in terms of making treatment decisions and predicting prognosis. Therefore, concordance between biopsy and the pathology results directly affects the prognosis of the patient. The results of our study demonstrated a $35.7 \%$ rate of discordance between Gleason scores obtained from transrectal prostate biopsy and RP surgical specimens. This rate brings into question the accuracy of the chosen treatment. Although numerous studies have investigated this issue, an effective way of reducing these discrepancies has yet to be determined. Further studies utilizing different imaging modalities and including larger sample sizes are needed.

\section{Ethics}

Ethics Committee Approval: The study was retrospectively reviewed by examining patient files. For this reason, ethical approval was not received.

Informed Consent: Retrospective study.

Peer-review: Externally peer-reviewed.

\section{Authorship Contributions}

Surgical and Medical Practices: E.Ö., T.N.Y., Concept: E.Ö., Design: E.Ö., T.N.Y., Data Collection or Processing: E.Ö., T.N.Y., Analysis or Interpretation: E.Ö., Literature Search: E.Ö., Writing: E.Ö.

Conflict of Interest: No conflict of interest was declared by the authors.

Financial Disclosure: The authors declared that this study received no financial support.

\section{References}

1. Ferlay J, Soerjomataram I, Dikshit R, et al. Cancer incidence and mortality worldwide: sources, methods and major patterns in GLOBOCAN 2012. Int J Cancer 2015;136:359-386.

2. Cohen MS, Hanley RS, Kurteva T, et al. Comparing the Gleason prostate biopsy and Gleason prostatectomy grading system: the Lahey Clinic Medical Center experience and an international metaanalysis. Eur Urol 2008;54:371-381.

3. Kuroiwa K, Shiraishi T, Naito S. Gleason score correlation between biopsy and prostatectomy specimens and prediction of high-grade Gleason patterns: significance of central pathologic review. Urology 2011;77:407-411.

4. Reis LO, Zani EL, Freitas LL, et al. Higher prostate weight is inversely associated with Gleason score upgrading in radical prostatectomy specimens. Adv Urol 2013;2013:710421.

5. Van Praet C, Libbrecht L, D'Hondt F, et al. Agreement of Gleason score on prostate biopsy and radical prostatectomy specimen: is there improvement with increased number of biopsy cylinders and the 2005 revised Gleason scoring? Clin Genitourin Cancer 2014; 12:160-166.

6. Gleason DF. Histologic grading of prostate cancer: a perspective. Hum Pathol 1992;23:273-279.

7. Kupelian P, Katcher J, Levin $\mathrm{H}$, et al. Correlation of clinical and pathologic factors with rising prostate-specific antigen profiles after radical prostatectomy alone for clinically localized prostate cancer. Urology 1996;48:249-260.

8. Epstein Jl, Allsbrook WC Jr, Amin MB, et al. ISUP Grading Committee: The 2005 International Society of Urological Pathology (ISUP) 
Consensus Conference on Gleason Grading of Prostatic Carcinoma. Am J Surg Pathol 2005;29:1228-1242.

9. Cookson MS, Fleshner NE, Soloway SM, et al. Correlation between Gleason score of needle biopsy and radical prostatectomy specimen: accuracy and clinical implications. J Urol 1997;157:559-562.

10. San Francisco IF, DeWolf WC, Rosen S, et al. Extended prostate needle biopsy improves concordance of Gleason grading between prostate needle biopsy and radical prostatectomy. J Urol 2003;169:136-140.

11. Stav K, Judith S, Merald H, et al. Does prostate biopsy Gleason score accurately express the biologic features of prostate cancer? Urol Oncol 2007;25:383-386.
12. Capitanio U, Karakiewicz PI, Valiquette L, et al. Biopsy core number represents one of foremost predictors of clinically significant Gleason sum upgrading in patients with low-risk prostate cancer. Urology 2009;73:1087-1091.

13. Donohue JF, Bianco FJ Jr, Kuroiwa K, et al. Poorly differentiated prostate cancer treated with radical prostatectomy: long-term outcome and incidence of pathological downgrading. J Urol 2006;176:991-995.

14. D'elia C, Cerruto MA, Cioffi A, et al. Upgrading and upstaging in prostate cancer: From prostate biopsy to radical prostatectomy. Mol Clin Oncol 2014;86:284-287. 